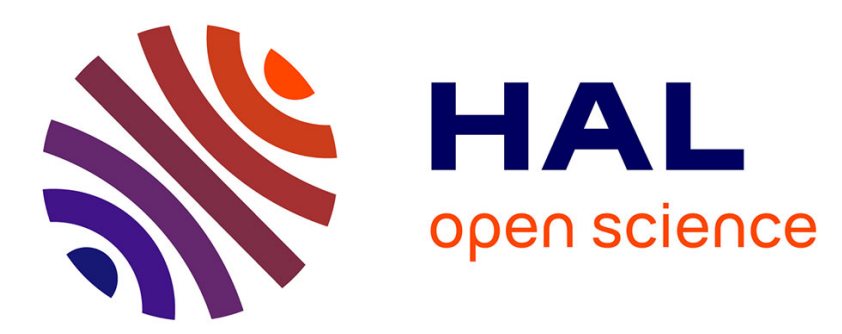

\title{
In-flight estimation of the aerodynamic characteristics of a Magnus effect-based airborne wind energy system
}

\author{
Eduardo Schmidt, Yashank Gupta, Jonathan Dumon, Ahmad Hably
}

\section{To cite this version:}

Eduardo Schmidt, Yashank Gupta, Jonathan Dumon, Ahmad Hably. In-flight estimation of the aerodynamic characteristics of a Magnus effect-based airborne wind energy system. REDEC 2018 International Conference on Renewable Energies for Developing countries (REDEC 2018), Nov 2018, Beyrouth, Lebanon. 10.1109/REDEC.2018.8598022 . hal-01895342

\section{HAL Id: hal-01895342 \\ https://hal.science/hal-01895342}

Submitted on 15 Oct 2018

HAL is a multi-disciplinary open access archive for the deposit and dissemination of scientific research documents, whether they are published or not. The documents may come from teaching and research institutions in France or abroad, or from public or private research centers.
L'archive ouverte pluridisciplinaire HAL, est destinée au dépôt et à la diffusion de documents scientifiques de niveau recherche, publiés ou non, émanant des établissements d'enseignement et de recherche français ou étrangers, des laboratoires publics ou privés. 


\title{
In-flight estimation of the aerodynamic characteristics of a Magnus effect-based airborne wind energy system
}

\author{
Eduardo Schmidt ${ }^{1}$, Yashank Gupta ${ }^{2}$, Jonathan Dumon ${ }^{2}$, and Ahmad Hably ${ }^{2}$
}

\begin{abstract}
Magnus effect-based Airborne wind energy (AWE) systems are a promising yet still unexplored concept for harnessing wind power at high-altitudes. While other aspects of the technology have been recently studied, the problem of obtaining accurate information regarding the aerodynamic behavior of the suspended cylinder as its spin ratio varies remains open. This paper presents an adaptation of an existing estimation strategy based on a constrained Extended Kalman filter (EKF) for the aerodynamic characterization of a smallscale Magnus effect-based AWE prototype. The evaluation is performed on data obtained during wind tunnel experiments, and results indicate that, after minor modifications, the chosen approach can indeed be applied to Magnus effect-based AWE systems. Moreover, provided that the cylinder's angular velocity is available, it can be employed for approximately determining the relationship existing between the aerodynamic coefficients of lift and drag and the spin ratio of the airborne structure.
\end{abstract}

\section{INTRODUCTION}

Airborne wind energy (AWE) systems aim at harvesting wind power by exploiting aerodynamic forces acting on lightweight suspended structures anchored to the ground by means of one or more tethers. Among other advantages, this technology is able to reach higher altitudes than conventional wind turbines, where the winds are generally stronger and more consistent, while considerably reducing construction and installation costs. Such characteristics allow AWE devices to be deployed potentially anywhere, even in regions where the wind would not be suitable for conventional turbines.

AWE systems usually consist of two major components, namely a ground station and an Airborne module (ABM), which are mechanically or sometimes even electrically connected by the tethers. Among the different concepts, one can distinguish ground-generation systems, which convert mechanical energy into electricity at the ground, and airbornegeneration systems, which generate electricity at the ABM and then transfer it to the ground through conducting tethers. Within these two groups there are many other possible subdivisions based on the type of ABM, steering concept, and the presence or absence of movement of the ground station. A comprehensive survey of the AWE technology can be found in [1].

Among these concepts, AWE systems based on the Magnus effect are ones that deserve attention due to their naturally stable configuration and high lift properties. The

\footnotetext{
${ }^{1}$ Federal University of Santa Catarina, Florianopolis, SC, Brazil edufschmidtegmail. com

$\begin{array}{cccll}{ }^{2} \text { Univ. } & \text { Grenoble } & \text { Alpes, } & \text { CNRS, } & \text { Greno- } \\ \text { ble INP, } & \text { GIPSA-lab, } & 38000 & \text { Grenoble, } & \text { France }\end{array}$
} yashank. guptadgipsa-lab.grenoble-inp. fr

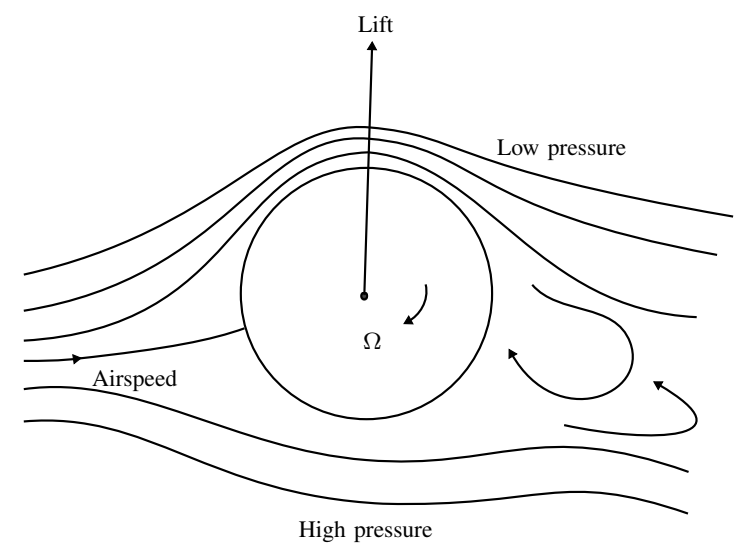

Fig. 1. The Magnus effect

Magnus effect is a phenomenon characterized by the appearance of a force on spinning spherical or cylindrical bodies as a result of their interaction with a fluid. The rotational movement of the body causes the fluid to be dragged faster around one side giving rise to a difference in pressure, which in turn leads to the so-called Magnus force directed towards the lower-pressure side, as indicated in Figure 1.

With respect to their constructive characteristics, Magnus effect-based AWE systems are comprised of a tethered cylinder capable of spinning around its longitudinal axis, as depicted in Figure 2. The tether traction resulting from the aerodynamic forces acting upon this cylinder is then used to drive the ground-generation systems. Because of the symmetry of the ABM, Magnus effect-based AWE devices are insensitive to the direction of the apparent wind, and thus more robust to wind gusts than other AWE concepts. Moreover, they also have a lift coefficient much higher than that of conventional wings - which can be increased even further by the addition of Thom discs to the cylinder [2] - and therefore be less sensitive to the effects of drag. Finally, a key characteristic of AWE devices which are based on the Magnus effect is that their aerodynamic coefficients do not depend on the angle-of-attack, as it happens with conventional airfoils. Instead, these coefficients are functions of another parameter known as spin ratio, which is the ratio between the angular velocity of the cylinder and the apparent wind velocity. As the angle-of-attack is usually hard to measure, and hence rather complex to control, getting rid of this variable can be very convenient, especially for power optimization approaches which require continuous ad- 
justment of the aerodynamic forces acting upon the system.

Since 2013, the Portuguese company Omnidea has been experimenting with a prototype in which the traction induced on the tether by the aerodynamic forces acting on a rotating cylindrical-balloon suspended by both aerostatic and aerodynamic lift mechanisms is used to move a ground-based generator and produce electricity, as described in [3], [4]. The feasibility of similar structures has been also studied in [5]. In [6], an approach is presented for the optimization of the vertical trajectories of a Magnus effect-based AWE system. In [7], a strategy based on the manipulation of both tether length and cycle period is presented for the control of the power produced by an AWE system with movement constrained to the vertical plane.

According to [8], in order to establish a control-oriented mathematical model for Magnus effect-based AWE systems, the determination of the aerodynamic coefficients of lift and drag of the ABM is a necessary step, which requires the study of flow past rotating cylinders in high Reynolds number regime. This is a very complicated phenomenon and, unfortunately, has not received enough attention from the aerodynamics community. Due to the magnitude of the effort required for a more comprehensive study involving e.g. a Computational fluid dynamics (CFD) analysis, the same work preferred to establish an approximation for the aerodynamic coefficients by analyzing data available in the literature for similar structures. Although useful for simulation, the results of this analysis are very unlikely to hold in experimental setups with actual prototypes. Therefore, an approach specific to Magnus effect-based AWE systems must still be devised for the determination of the lift and drag coefficients as functions of the cylinder's spin ratio.

In [9], a filtering strategy for AWE systems based on an EKF inspired by [10] is presented. As a major difference from previous works, this approach utilizes the perfect measurement strategy described in [11] for the enforcement of an orthogonality constraint between the lift force and the apparent wind vectors. By jointly estimating the wind conditions at the flight level and the aerodynamic forces of lift and drag, it is then able to compute variables such as the equivalent aerodynamic efficiency of the system, as demonstrated in both simulation and field experiments with a small-scale prototype.

Motivated by the importance of obtaining information about the lift and drag coefficients of spinning cylinders immersed in fluid, and considering the current lack of solutions available for determining these variables, this paper adapts the approach presented in [9] for the estimation of the aerodynamic parameters.

The paper is organized as follows. Section II gives a brief mathematical description of a Magnus effect-based AWE system. Section III presents the EKF proposed for the estimation of the aerodynamic characteristics of the Magnus cylinder. Section IV presents both a test setup comprising a small-scale AWE prototype operating in a wind tunnel and the estimation results obtained with experimental data. Finally, Section V discusses the results, and concludes regarding the suitability of the estimation approach.

Regarding notation, vectors are represented by bold characters, their Euclidean norm by $\|$.$\| , and scalar values by$ non-bold characters. In situations involving more than one reference frame, the frame in which a vector is expressed is indicated by a subscript. The symbol $x_{k}$ indicates the $k$-th sample of a discrete signal sampled from an originally continuous quantity $x$. Otherwise explicitly noted, all quantities are described in the International system of units (SI).

\section{SYSTEM DESCRIPTION}

\section{A. System Model}

This paper considers a Magnus effect-based AWE system consisting of spinning cylinder mounted on a rigid frame and tethered to a ground station, as depicted in Figure 2.

The operation of the Magnus effect-based AWE system under consideration comprises two main phases: the active (generation, reel-out) phase and the passive (retraction, reelin) phase. In the generation phase, the Magnus cylinder follows a predefined trajectory, producing a high tension in the tether while simultaneously reeling it out from the drum coupled to a generator on the ground. In the retraction phase, this process is reversed, and the generator is used as a motor to reel the tether back in. Through the performance of a low-traction maneuver during the passive phase, the AWE system is expected to consume only a fraction of the energy produced within the first half of the pumping cycle.

As presented in [8], the dynamic equations of a Magnus cylinder can be derived in the cylinder's body frame, and then transferred to an inertial frame centered at the ground station through the application of rotation matrices. The position vector of the Magnus cylinder in the body frame $\mathbf{r}_{b}$ is given by

$$
\mathbf{r}_{b}=\left(R_{b}^{i}\right)\left(L_{f l i p}\right) \mathbf{r}_{i}
$$

where, $\mathbf{r}_{i}$ represents the position vector in the inertial frame, and $R_{b}^{i}$ and $L_{\text {flip }}$ denote, respectively, the rotation matrix between the body and the inertial frames and the matrix used to flip the inertial coordinate system at the ground in order to align it with the conventional North-East-Down (NED) frame. Hence, the equation of translational motion of the Magnus effect-based AWE system is given by

$$
\dot{\mathbf{r}_{\mathbf{i}}}=\left(L_{f l i p}\right)^{-1}\left(R_{b}^{i}\right)^{-1} \mathbf{v}_{b}
$$

The equation describing the rate of change of the cylinder's translational velocity is obtained from Newton's second law of motion and from Coriolis theorem, and is given by

$$
\dot{\mathbf{v}}_{b}=\frac{1}{m_{e q}}\left(\mathbf{F}_{b}-\tilde{\omega} \mathbf{v}_{b}\right)
$$

where

$$
\tilde{\omega}=\left[\begin{array}{ccc}
0 & -r & q \\
r & 0 & -p \\
-q & p & 0
\end{array}\right]
$$


The variables $p, q$, and $r$ represent the angular rates about the $x_{b}, y_{b}$, and $z_{b}$ body-frame axes, respectively, whereas $\mathbf{F}_{b}$ corresponds to the sum of all forces acting on the Magnus cylinder. It is expressed as

$$
\mathbf{F}_{b}=\mathbf{F}_{L}+\mathbf{F}_{D}+\mathbf{F}_{d y}+\mathbf{W}_{b}+\mathbf{F}_{b u}+\mathbf{F}_{r}
$$

where $\mathbf{F}_{L}$ and $\mathbf{F}_{D}$ represent the lift and drag forces in the $x_{b} z_{b}$ plane, and $\mathbf{F}_{d y}$ is the drag force in the $y_{b}$ direction. These forces are given by

$$
\begin{gathered}
\mathbf{F}_{L}=\frac{1}{2} \rho S_{c y l} v_{a x z}^{2} C_{L} \mathbf{e}_{F l} \\
\mathbf{F}_{D}=\frac{1}{2} \rho S_{c y l} v_{a x z}^{2} C_{D} \mathbf{e}_{F d} \\
\mathbf{F}_{d y}=\frac{1}{2} \rho S_{c y l} v_{a y}^{2} C_{d y} \mathbf{y}_{b}
\end{gathered}
$$

where $S_{c y l}$ refers to the projected surface area of the cylinder, $v_{a x z}$ and $v_{a y}$ to the components of the apparent wind velocity, and $C_{L}$ and $C_{D}$ represent the coefficients of lift and drag, respectively. Still in accordance with [8], $\mathbf{W}_{b}, \mathbf{F}_{b u}$, and $\mathbf{F}_{r}$ are used to denote the weight in the $z_{b}$ direction, the buoyancy force, and the tether traction force evaluated at the ground station, respectively. For the sake of simplicity, the tether dynamics is neglected, meaning that effects due to its drag, elasticity, and inertia are not considered.

In order to simulate the dynamics of Magnus effectbased AWE systems, it is necessary to understand how the aerodynamic coefficients $C_{L}$ and $C_{D}$ vary with the spin ratio parameter $X$, defined as

$$
X=\frac{\omega_{c y l} r_{c y l}}{\left\|v_{a}\right\|}
$$

with $\omega_{c y l}$ being the angular velocity of the cylinder, $r_{c y l}$ its radius, and $\left\|\mathbf{v}_{a}\right\|$ the magnitude of the apparent wind velocity. In [8], the relationship between the aerodynamic coefficients and the spin ratio is given by polynomial expressions, as proposed in [6].

\section{ESTIMATION STRUCTURE}

A discrete time EKF similar to that proposed in [9] was implemented and utilized for estimating the aerodynamic forces acting upon the system, as well as other important variables. The state vector $\mathbf{x}$ of the filter is given by

$$
\mathbf{x}=\left[\mathbf{r}^{T}, \dot{\mathbf{r}}^{T}, \ddot{\mathbf{r}}{ }^{T}, \mathbf{V}_{w}^{T}, \mathbf{F}_{L}^{T}, F_{D}, F_{r}\right]^{T},
$$

and contains the position $\mathbf{r}$, the velocity $\dot{\mathbf{r}}$, and the acceleration $\ddot{\mathbf{r}}$ of the equivalent point-mass representing the ABM. Moreover, it also includes the nominal wind $\mathbf{V}_{w}$, the lift force $\mathbf{F}_{L}$, the magnitudes of the equivalent drag force $F_{D}-$ which also comprises tether drag effects - and of the tether traction force $F_{r}$.

The evolution of these states in time is ruled by a simplified dynamical model similar to those presented in [9] and [8], and which can be described in discrete time by the following set of difference equations

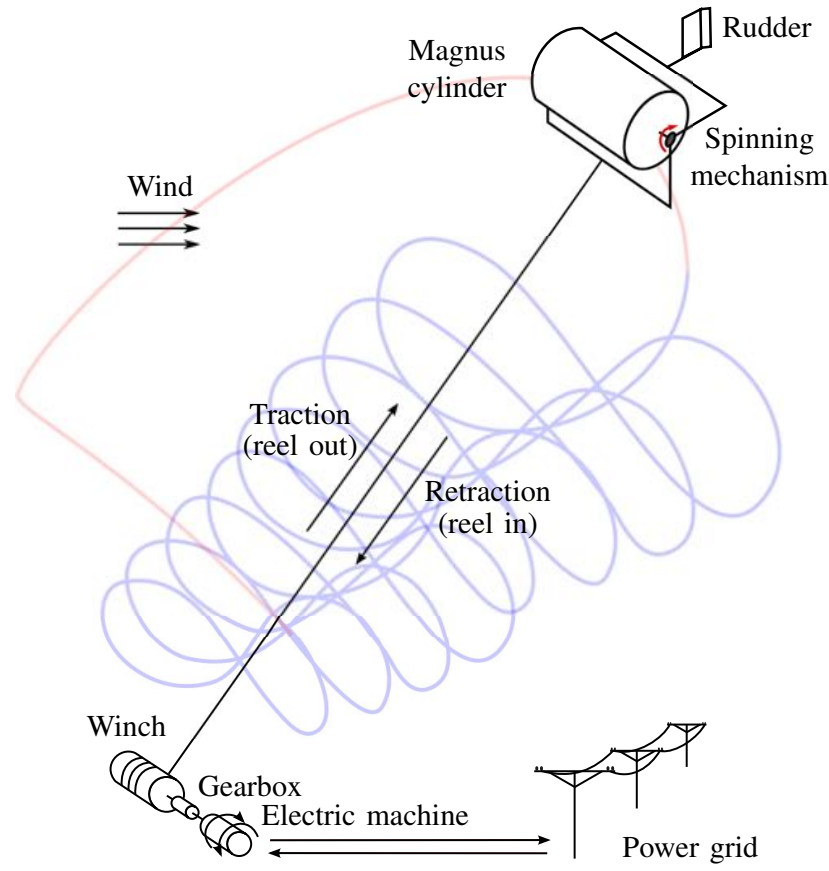

Fig. 2. Conceptual representation of a Magnus effect-based AWE system in a pumping-kite configuration during the active (blue) and the passive (red) phases.

$$
\begin{aligned}
\mathbf{r}_{k+1} & =\mathbf{r}_{k}+\dot{\mathbf{r}}_{k} T_{s} \\
\dot{\mathbf{r}}_{k+1} & =\dot{\mathbf{r}}_{k}+\ddot{\mathbf{r}}_{k} T_{s} \\
\ddot{\mathbf{r}}_{k+1} & =\left(1 / m_{e q}\right)\left(\mathbf{F}_{L k}+\mathbf{F}_{D k}+\mathbf{F}_{g_{k}}+\mathbf{F}_{b u k}+\mathbf{F}_{r k}\right) \\
\mathbf{V}_{w k+1} & =\mathbf{V}_{w k} \\
\mathbf{F}_{L k+1} & =\mathbf{F}_{\mathbf{L} k} \\
F_{D k+1} & =F_{D k} \\
F_{r k+1} & =F_{r k}
\end{aligned}
$$

where $F_{g}$ is the weight of the equivalent point-mass in the inertial frame and $T_{s}$ is the sampling period. Regarding the observed variables, it is assumed that measurements of both the position and velocity vectors of the ABM, r and $\dot{\mathbf{r}}$, respectively, are available. Moreover, the magnitude of the tether traction $F_{r}$, as well as the nominal wind speed $V_{w}$ and direction $\phi_{w}$ are too assumed measurable quantities. This choice of observations took into consideration characteristics of the small-scale prototype available in the lab, as will be discussed in Section IV, and led to an observation vector given by

$$
\mathbf{y}=\left[\mathbf{r}^{T}, \dot{\mathbf{r}}^{T}, V_{w}, \phi_{w}, F_{r}, \delta\right]^{T},
$$

Regarding the observation $\delta$, it does not model any physical quantity. It is, in fact, a made-up variable representing the inner product between the lift vector and the apparent wind $\mathbf{v}_{a}$, i.e. $\delta=\mathbf{F}_{L}^{T} \mathbf{v}_{a}$, and is included in the filtering as a way to enforce the orthogonality constraint between these two 


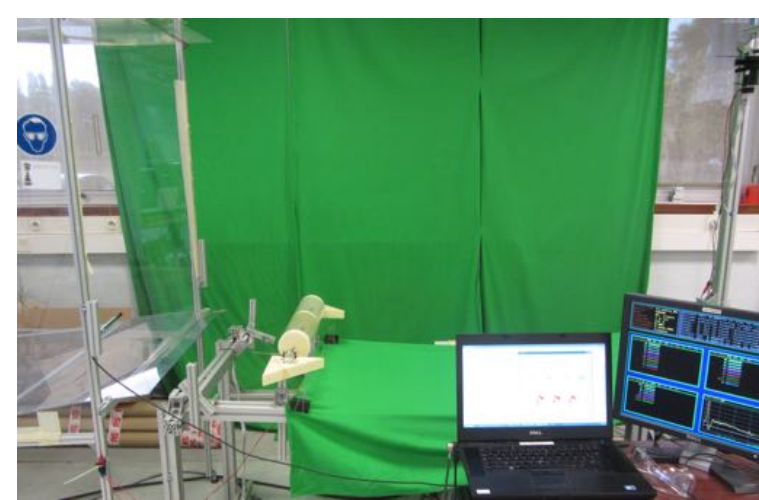

Fig. 3. The Matlab/Simulink real-time setup used in the experiments.

vectors according to the perfect measurement technique. The inclusion of such a constraint in the form of a measurement is a novel practice in AWE, being first reported in [9].

All derivatives required for propagating the state and the covariance matrices in the Kalman algorithm are numerically computed using forward finite differences, and therefore analytic expressions for the Jacobians of the dynamic equations and of the observation functions are not necessary.

\section{EXPERIMENTAL RESULTS}

A small-scale experimental setup built at GIPSA-Lab and previously used in [12], [13], [7], [14] was employed to validate the proposed estimation approach. This setup comprises a custom wind tunnel, a Magnus cylinder, and a ground station equipped with a number of instruments for the measurement of different flight parameters.

\section{A. Wind tunnel}

The wind tunnel was built to allow for the realization of experiments independently of weather conditions. It consists of nine $800 \mathrm{~W}$ brushless motors evenly distributed over a $1.2 \mathrm{~m}^{2}$ surface, and capable of generating an air flow of up to $9 \mathrm{~m} / \mathrm{s}$. The air speed is measured by a hot wire anemometer at a rate of $1 \mathrm{~Hz}$ through a serial interface, and the controllers are deployed on top of the XPC real-time toolbox for Matlab.

\section{B. Ground station}

The ground station consists of a Maxon 2260L DC 100W dynamo-motor system driven by an ADS 50/10 4 quadrants amplifier manufactured by the same company. The length and elevation of the tethers are measured by two incremental encoders mounted on the ground station. Tether tension is acquired using a rotative torque sensor, and the PCI DAS1200 Digital-to-analog converter (DAC) module is employed to communicate and send control references to the actuation drivers.

\section{Magnus cylinder}

A lightweight Magnus cylinder built with carbon rods, polystyrene, and transparent plastic paper was used as the $\mathrm{ABM}$ in the experiments, as depicted in Figure 5, which

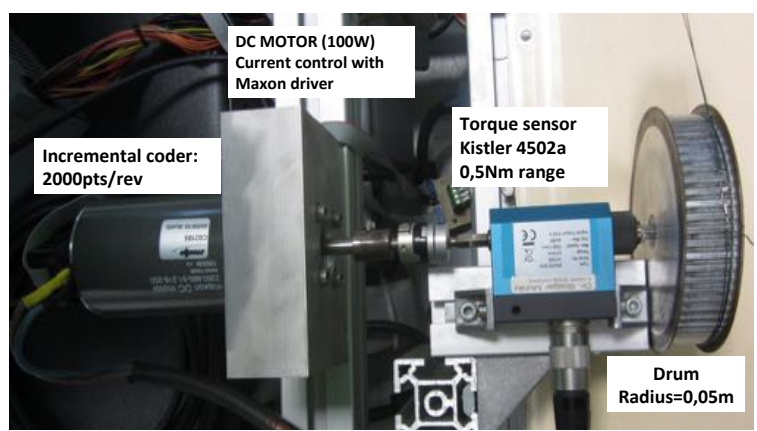

Fig. 4. The Ground station to which the Magnus cylinder was tethered.

TABLE I

PARAMETERS OF THE MAGNUS CYLINDER

\begin{tabular}{lll}
\hline Symbol & Description & Value \\
\hline$M_{\mathrm{Mag}}$ & Magnus cylinder's mass & $0.11 \mathrm{Kg}$ \\
$M I$ & Tether's linear mass & $0 \mathrm{Kg} / \mathrm{m}$ (neglected) \\
$R$ & Magnus cylinder's radius & $0.047 \mathrm{~m}$ \\
$L$ & Magnus cylinder's length & $0.45 \mathrm{~m}$ \\
$M_{I M}$ & Rotor's mass & $0.0481 \mathrm{Kg}$ \\
$\rho$ & Air density & $1.225 \mathrm{Kg} / \mathrm{m}^{3}$ \\
\hline
\end{tabular}

also portrays the mini DC motor responsible for spinning the airborne structure. Custom hardware was employed for measuring and controlling the angular speed of the cylinder, whose parameters are presented in Table I.

Utilizing this small-scale setup, a series of experiments were performed in which the Magnus cylinder was exposed to varying wind conditions, such as those depicted in Figure 7. With the ABM following trajectories similar to that of Figure 6, which comprise reel in and reel out cycles, and with its angular velocity $\omega$ being continuously varied, several variables including the tether length $l$, its elevation angle $\beta$, and traction force $F_{r}$ were recorded.

Based on these data, observation vectors were computed for each time instant according to Equation 12, and later used to feed the EKF and produce the results hereafter presented for a $100 \mathrm{~s}$ window.

Figure 7, presents the air speed over the course of the ex-

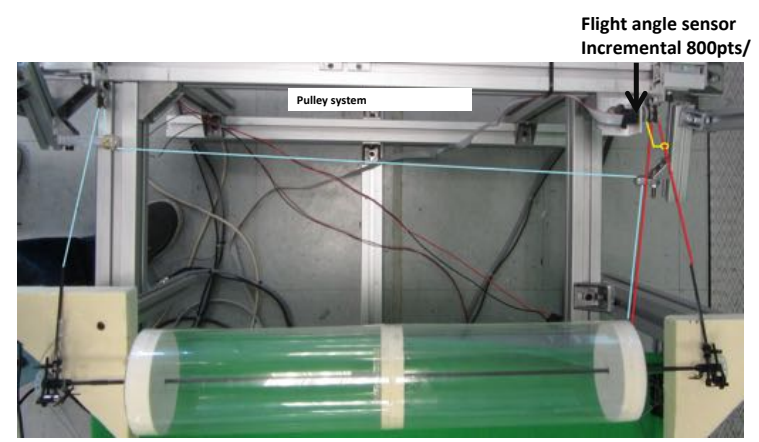

Fig. 5. The Magnus cylinder used as the ABM in the wind tunnel experiments. 


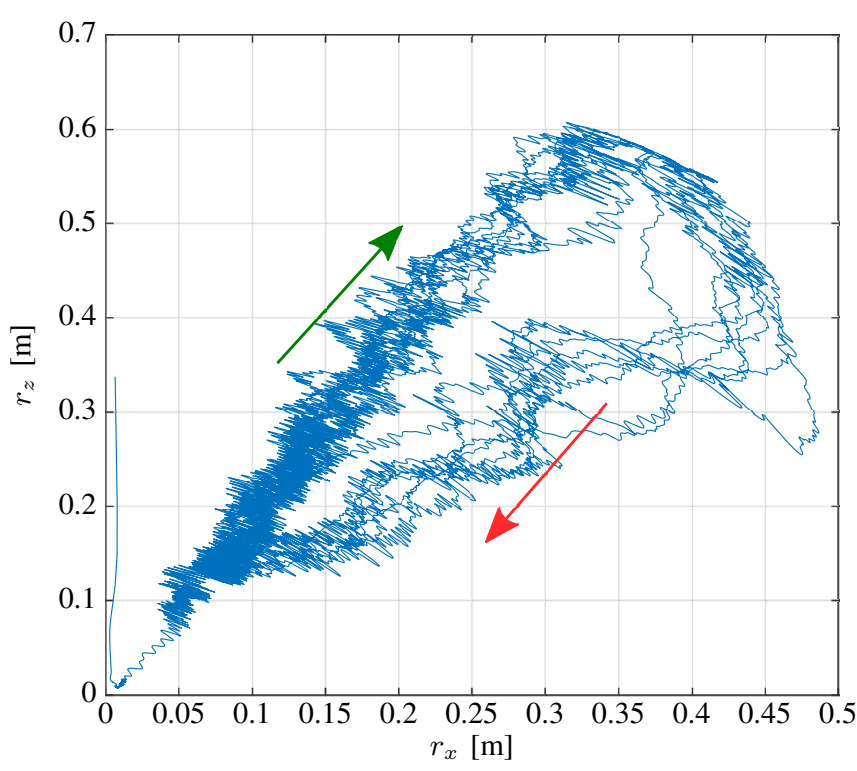

Fig. 6. Flight trajectory of the small-scale Magnus cylinder on the vertical plane

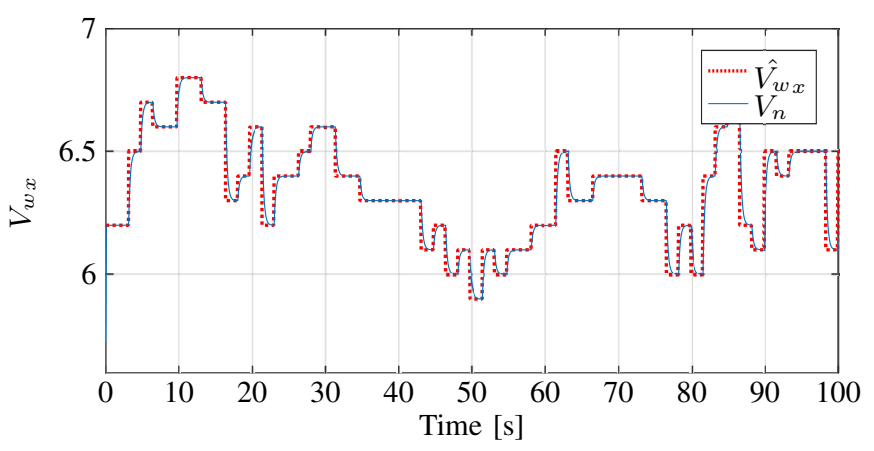

Fig. 7. Wind speed used as reference for the wind tunnel and estimates obtained from the filter

periment, as measured by the hot wire anemometer within the wind tunnel, alongside the wind speed estimates produced by the filter. It can be observed that the curve corresponding to the estimated wind speed exhibits a non-negligible dynamic when compared to the signal output by the wind sensor, which corresponds to the estimator's dynamic.

The filter estimates obtained for the considered time interval also allowed for the computation of the cylinder's spin ratio, as well as of the lift and equivalent drag coefficients according to equations 9, 6, and 7. Finally, Figures 9 and 10 present the evolution for several pumping cycles of the estimated $C_{L}$ and $C_{D}$ parameters, respectively, and show that these quantities assume values coherent to other results reported in the literature for Magnus cylinders, changing with variations in the spin ratio, as expected.

Another set of tests dedicated to the identification of the Magnus cylinder's aerodynamic characteristics for a broader range of spin ratios was also performed. In these

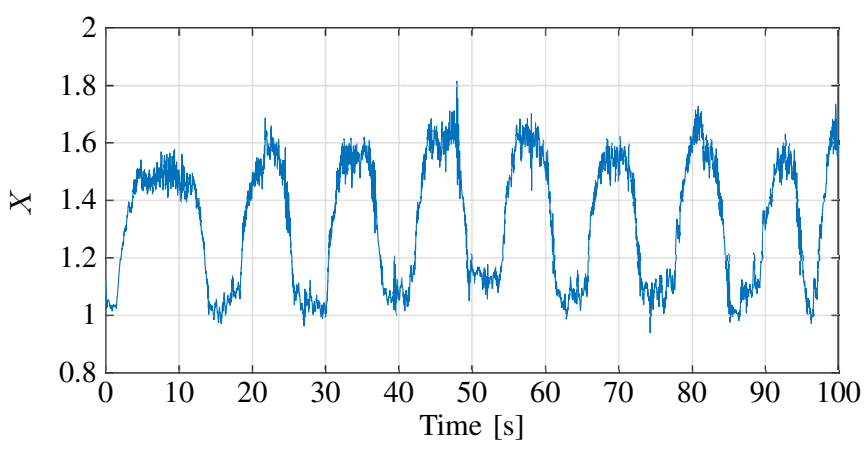

Fig. 8. Estimated spin ratio of the Magnus cylinder

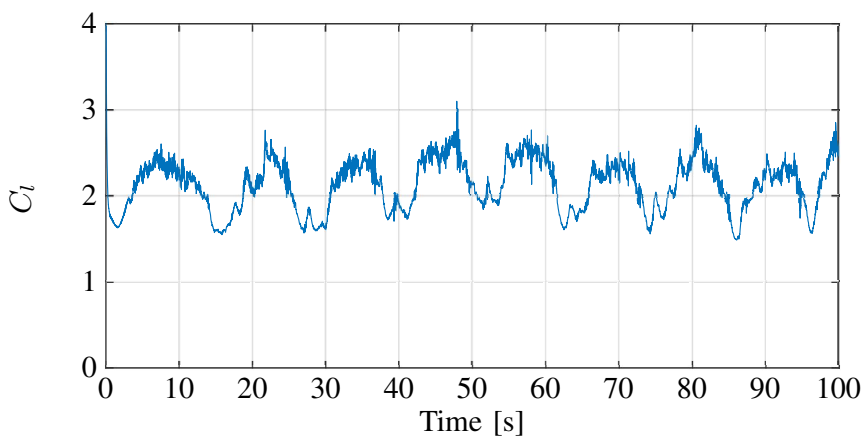

Fig. 9. Estimated lift coefficient of the system

experiments, the angular velocity $\omega$ was continuously varied between $150 \mathrm{rad} / \mathrm{s}$ and $225 \mathrm{rad} / \mathrm{s}$, while the mean wind speed produced by the wind tunnel oscillated between $4.5 \mathrm{~m} / \mathrm{s}$ and $7 \mathrm{~m} / \mathrm{s}$, resulting in spin ratio values between 1 and 2.5. The tether length $l$ was increased slowly during the experiments to ensure that the dry friction force $\Gamma_{s}$ in the pulley system always acted in the same direction for all data sets. In order to mitigate the influence of this effect, the magnitude of the dry friction force, identified in [14] as approximately $\Gamma_{s}=0.4 \mathrm{~N}$ was finally removed from the tether traction measurements, resulting in

$$
F_{r_{k}}^{\prime}=F_{r_{k}}+\Gamma_{s}
$$

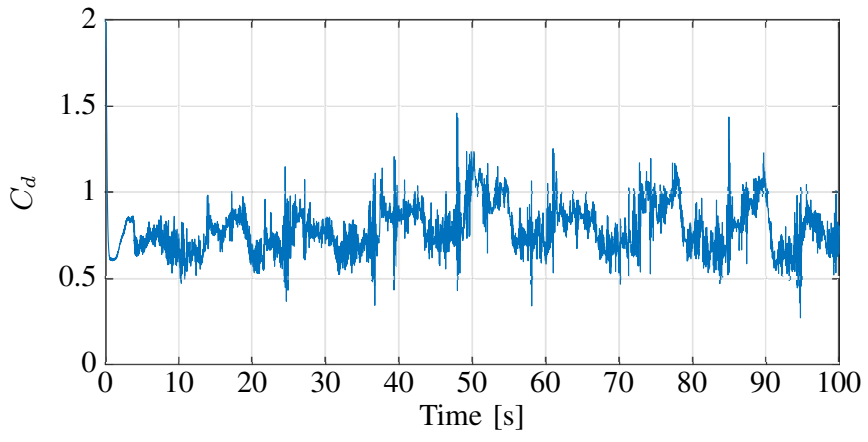

Fig. 10. Estimated drag coefficient of the system 
where $\mathbf{F}_{r_{k}}^{\prime}$ corresponds to the tether traction after the dry friction compensation, which was then used to feed the EKF.

In Figure 11, the aerodynamic coefficients estimated during the aforementioned identification experiments and also during the pumping cycles illustrated in Figure 6 are compared with those predicted for the same range of spin ratios by the polynomial model presented in [6] and given by

$$
\begin{aligned}
& C_{L}=0.0126 X^{4}-0.2004 X^{3}+0.7482 X^{2}+1.3447 X \\
& C_{D}=-0.0211 X^{3}+0.1873 X^{2}+0.1183 X+0.5
\end{aligned}
$$

Except for an offset of 0.4 , the $C_{L}$ values estimated during the identification experiments follow the same trend of the polynomial model for all considered spin ratio values. This does not happen for the $C_{D}$ estimates, which present a behavior different from that predicted by the polynomial model for spin ratios larger than 1.5. This phenomenon can be explained by the effects on the drag coefficient of the tethers, mounting frame, and spinning mechanism not considered in the model proposed in [6], which assumes a single, theoretically endless cylinder. The results indicate that, in order to properly account for the influence of these structures on the drag characteristics of the ABM, another model might have to be considered.

Regarding the $C_{L}$ and $C_{D}$ values estimated during the pumping cycles shown in Figure 6, it can be observed that they present a slightly different behavior. These discrepancies can be attributed to the dry friction present in the system but not accounted for. In the traction phase, while the tether is being reeled out and the spin ratio is kept at around $X=1.5$, the dry friction effects lead to an underestimation of $C_{L}$ and $C_{D}$, since it causes a decrease of the measured tether traction. On the other hand, in the retraction phase while which the spin ratio oscillates around $X=1.1$, the dry friction is responsible for increasing the measured tether traction, hence leading to and overestimation of the aerodynamic coefficients, as can be clearly observed in Figure 11. It, therefore, makes sense to consider the two phases of the pumping cycle independently. Moreover, the fact that the overestimation for low spin ratios is less than the underestimation for high spin ratios suggests that dry friction in the system depends on the traction force itself.

\section{CONCLUSION}

The EKF approach used in this paper has provided an important insight into the behavior of the aerodynamic coefficients of Magnus cylinders. The values estimated for $C_{L}$ and $C_{D}$ support our initial understanding of the aerodynamic properties, and provide means of obtaining improved aerodynamic models for the analysis of Magnus effect-based AWE systems.

One advantage of the proposed method is that the identification of the aerodynamic model can be done in flight with the same set of sensors already used to monitor and control the system, eliminating the need of other high-end wind and force measurement measuring devices.

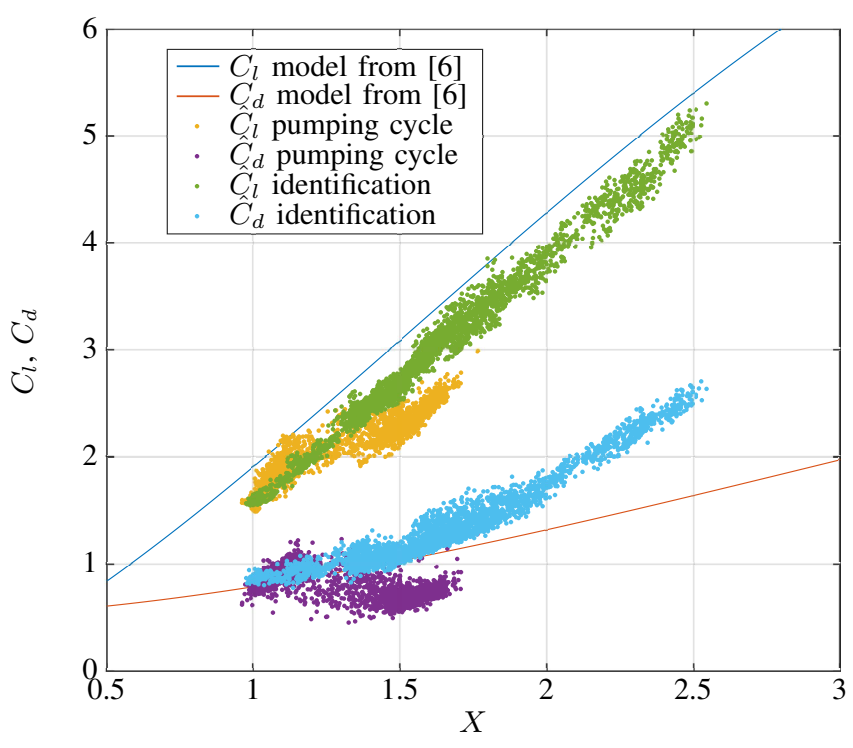

Fig. 11. Comparison of the lift and equivalent drag coefficients estimated during the pumping cycles and identification experiments with the values predicted by the polynomial model proposed in [6].

Besides, an approach based on the EKF also allows for real-time estimation of the aerodynamic characteristics of the system using off-the-shelf embedded hardware, and paves the way for the utilization of more sophisticated control mechanisms which rely on these data.

Finally, the dry friction effects noticed in the small-scale prototype used in the experiments, which has four pulleys and two tethers is not expected to affect larger systems with a single line, and therefore should not be seen as a significant problem.

\section{REFERENCES}

[1] A. Cherubini, A. Papini, R. Vertechy, and M. Fontana, "Airborne wind energy systems: A review of the technologies," Renewable and Sustainable Energy Reviews, vol. 51, pp. 1461-1476, 2015.

[2] A. Thom, S. Sengupta, and J. Cormack, Air torque on a cylinder rotating in an air stream. 1933.

[3] R. J. Penedo, T. C. Pardal, P. M. S. Silva, N. M. Fernandes, and T. R. C. Fernandes, "High altitude wind energy from a hybrid lighterthan-air platform using the magnus effect," in Airborne Wind Energy, pp. 491-500, Springer, 2013.

[4] P. Tiago and S. Pedro, "Analysis of experimental data of a hybrid system exploiting the magnus effect for energy from high altitude wind," in Book of Abstracts of the Airborne Wind Energy Conference, 2015.

[5] L. Perković, P. Silva, M. Ban, N. Kranjčević, and N. Duić, "Harvesting high altitude wind energy for power production: The concept based on magnus effect," Applied energy, vol. 101, pp. 151-160, 2013.

[6] M. Milutinović, M. Čorić, and J. Deur, "Operating cycle optimization for a magnus effect-based airborne wind energy system," Energy conversion and management, vol. 90, pp. 154-165, 2015.

[7] A. Hably, J. Dumon, and G. Smith, "Control of an airborne wind energy system with a magnus effect," in American Control Conference (ACC), 2016, pp. 4978-4983, IEEE, 2016.

[8] Y. Gupta, J. Dumon, and A. Hably, "Modeling and control of a magnus effect-based airborne wind energy system in crosswind maneuvers," in The 20th World Congress of the International Federation of Automatic Control, 2017. 
[9] R. Saraiva, M. D. Lellis, E. Schmidt, and A. Trofino, "Dynamics identification, filtering and control design for power kites," IFACPapersOnLine, vol. 50, no. 1, pp. 11938 - 11943, 2017. 20th IFAC World Congress.

[10] M. Ranneberg, "Sensor setups for state and wind estimation for airborne wind energy converters," arXiv preprint arXiv:1309.1029, 2013.

[11] D. Simon, "Kalman filtering with state constraints: a survey of linear and nonlinear algorithms," IET Control Theory \& Applications, vol. 4, no. 8, pp. 1303-1318, 2010.

[12] A. Hably, R. Lozano, Alamir, and J. M. Dumon, "Observer-based control of a tethered wing wind power system: indoor real-time experiment," in The 2013 American Control Conference, (ACC2013). 2013

[13] R. Lozano, J. Dumon, A. Hably, and M. Alamir, "Energy production control of an experimental kite system in presence of wind gusts," in 2013 IEEE/RSJ International Conference on Intelligent Robots and Systems (IROS), pp. 2452-2459, IEEE, 2013.

[14] A. Hably, J. Dumon, G. Smith, and P. Bellemain, Control of a Magnus Effect-Based Airborne Wind Energy System. in book Airborne Wind Energy: Advances in Technology Development and Research Springer, 2018. 